\title{
SUPPLEMENTARY TABLE S1:
}

\begin{tabular}{|c|c|c|c|c|c|}
\hline No. & $\begin{array}{c}\text { Gene } \\
\text { name } \\
\text { (Variant) }\end{array}$ & $\begin{array}{l}\text { Gene } \\
\text { ID }\end{array}$ & Primer Sequences $\left(5-3^{\prime}\right) \dagger$ & $\begin{array}{l}\text { Primer } \\
\text { length } \\
\text { (bases) }\end{array}$ & $\begin{array}{l}\text { PCR } \\
\text { product } \\
\text { length } \\
\text { (basepairs) }\end{array}$ \\
\hline 1 & $\begin{array}{l}L D L R \\
\text { (ins242G) }\end{array}$ & 3949 & $\begin{array}{l}\text { F: Biotin-5'-tga cag ttc aat cet gtc tct tct } \\
\text { g-3', } \\
\text { R: } 5^{\prime} \text {-ata gca aag gca ggg cca cac tta-3', }\end{array}$ & $\begin{array}{l}25 \\
24\end{array}$ & 175 \\
\hline 2 & $\begin{array}{l}L D L R \\
\text { (ins397G) }\end{array}$ & 3949 & $\begin{array}{l}\text { F: Biotin-5'-gcg gtc cca aaa ggg tca gt- } \\
\text { gtg gtc tcg gcc atc cat cce tgc-3' } \\
\text { R: 5'-ttc taa tac gac tca cta tag-acg ccc } \\
\text { cgc ccc cac cct gcc ccg-3' }\end{array}$ & 44 & 519 \\
\hline 3 & $\begin{array}{l}L D L R \\
(\mathrm{E} 387 \mathrm{~K})\end{array}$ & 3949 & $\begin{array}{l}\text { F: 5'-ttc taa tac gac tea cta tag-ctg acc } \\
\text { tcg ctc ccc gga cc-3' }\end{array}$ & 41 & 577 \\
\hline 4 & $\begin{array}{l}\text { LDLR } \\
\text { (L393K) }\end{array}$ & 3949 & $\begin{array}{l}\text { R: Biotin-5'-gcg gtc cca aaa ggg tca gt- } \\
\text { gcc ctc agc gtc gtg gat acg-3' }\end{array}$ & 41 & \\
\hline 5 & $\begin{array}{l}L P L \\
(\mathrm{D} 9 \mathrm{~N})\end{array}$ & 4023 & $\begin{array}{l}\text { F: Biotin-5'-geg gtc cca aaa ggg tca gt- } \\
\text { ctc ata tcc aat ttt tcc tt-3' } \\
\text { R: } 5^{\prime}-\text { ttc taa tac gac tca cta tag-ctc ttc } \\
\text { ccc aaa gag cct cc-3' }\end{array}$ & 40 & 252 \\
\hline
\end{tabular}




\begin{tabular}{|c|c|c|c|c|c|}
\hline 6 & $\begin{array}{l}L P L \\
(\mathrm{~N} 291 \mathrm{~S})\end{array}$ & 4023 & $\begin{array}{l}\text { F: } 5 \text { '-ttc taa tac gac tca cta tag-gcc gag } \\
\text { ata caa tct tgg tg-3' } \\
\text { R: Biotin-5'-gcg gtc cca aaa ggg tca gt- } \\
\text { gca tga tga aat agg act-3' }\end{array}$ & 41 & 334 \\
\hline 7 & $\begin{array}{l}\text { CETP } \\
(\mathrm{I} 405 \mathrm{~V})\end{array}$ & 1071 & $\begin{array}{l}\text { F: 5'-ttc taa tac gac tca cta tag-gga atg } \\
\text { ggc tag caa tcc tgg-3' } \\
\text { R: Biotin-5'-gcg gtc cca aaa ggg tca gt- } \\
\text { tga aat ggg aag ctc tgt cag-3' }\end{array}$ & 42 & 38 \\
\hline 8 & $\begin{array}{l}\text { CETP } \\
\text { (D442G) }\end{array}$ & 1071 & $\begin{array}{l}\text { F: 5'-ttc taa tac gac tca cta tag-gge tct } \\
\text { gtc ccc tcg ccc agg-3' } \\
\text { R: Biotin-5'-gcg gtc cca aaa ggg tca gt- } \\
\text { gct ttg tac tct cat ctc gag-3' }\end{array}$ & 41 & 160 \\
\hline 9 & $\begin{array}{l}L I P C \\
(\mathrm{C}-514 \mathrm{~T})\end{array}$ & 3990 & $\begin{array}{l}\text { F: Biotin-5'-geg gtc cca aaa ggg tca gt- } \\
\text { aag aag tgt gtt tac tct agg atc-3' } \\
\text { R: } 5^{\prime} \text {-ttc taa tac gac tca cta tag-tgg ctt } \\
\text { cca cgt ggc tgc cta ag-3' }\end{array}$ & 44 & 340 \\
\hline 10 & $\begin{array}{l}F G B \\
(\mathrm{G}-455 \mathrm{~A})\end{array}$ & 2244 & $\begin{array}{l}\text { F: Biotin-5'-geg gtc cca aaa ggg tca gt- } \\
\text { ggg tat gaa ttt gtt att ttg-3' } \\
\text { R: } 5^{\prime} \text { ttc taa tac gac tca cta tag-ggc aac } \\
\text { cac taa aat cgt gac } t-3 \text { ' }\end{array}$ & 41 & 219 \\
\hline
\end{tabular}




\begin{tabular}{|c|c|c|c|c|c|}
\hline 11 & \begin{tabular}{|l} 
ITGB3 \\
(L33P)
\end{tabular} & 3690 & $\begin{array}{l}\text { F: Biotin-5'-gcg gtc cca aaa ggg tca gt- } \\
\text { ttg ctg gac ttc tct ttg-3' } \\
\text { R: } 5 \text { '-ttc taa tac gac tca cta tag-ctc act } \\
\text { ggg aac tcg atg g-3', }\end{array}$ & 38 & 168 \\
\hline 12 & $\begin{array}{l}A G T \\
(\mathrm{M} 235 \mathrm{~T})\end{array}$ & 183 & $\begin{array}{l}\text { F: } 5 \text { '-ttc taa tac gac tea cta tag-gga tgc } \\
\text { gca caa ggt cct gtc-3' } \\
\text { R: Biotin-5'-gcg gtc cca aaa ggg tca gt- } \\
\text { aca tgg ata cta agt cct agg gc-3' }\end{array}$ & 42 & 423 \\
\hline 13 & $\begin{array}{l}\text { AGTR1 } \\
\text { (A1166C) }\end{array}$ & 185 & $\begin{array}{l}\text { F: 5'-ttc taa tac gac tca cta tag-atg agc } \\
\text { acg ctt tcc tac cgc-3' } \\
\text { R: Biotin-5'-gcg gtc cea aaa ggg tca gt- } \\
\text { aag cag gct agg gag att gca ttt c-3' }\end{array}$ & $\begin{array}{l}42 \\
45\end{array}$ & 415 \\
\hline 14 & $\begin{array}{l}\text { NOS3 } \\
\text { (T-786C) }\end{array}$ & 4846 & $\begin{array}{l}\text { F: 5'-ttc taa tac gac tca cta tag-atg ctc } \\
\text { cca cca ggg cat-3' } \\
\text { R: Biotin-5'-gcg gtc cca aaa ggg tca gt- } \\
\text { gtc ctt gag tct gac att-3' }\end{array}$ & 39 & 277 \\
\hline 15 & $\begin{array}{l}\text { NOS3 } \\
\text { (E298D) }\end{array}$ & 4846 & $\begin{array}{l}\text { F: 5'-ttc taa tac gac tca cta tag-cat gag } \\
\text { gct cag ccc cag-3' } \\
\text { R: Biotin-5'-geg gtc cca aaa ggg tca gt- } \\
\text { agt caa tcc ctt tgg tgc-3' }\end{array}$ & 39 & 247 \\
\hline
\end{tabular}




\begin{tabular}{|c|c|c|c|c|c|}
\hline 16 & $\begin{array}{l}\text { CBS } \\
(\mathrm{T} 833 \mathrm{C})\end{array}$ & 875 & $\begin{array}{l}\text { F: } 5^{\prime}-\text { ttt tgc tgg cet tga gec ctg aa-3', } \\
\text { R: Biotin-5'-ggc cgg gct ctg gac tc-3', }\end{array}$ & $\begin{array}{l}23 \\
17\end{array}$ & 189 \\
\hline 17 & $\begin{array}{l}\text { MTR } \\
\text { (A2756G) }\end{array}$ & 4548 & $\begin{array}{l}\text { F: 5'-cca ggc agg aat tag cac agt tg-3' } \\
\text { R: Biotin-5'-ttg aac tag aag aca gaa att } \\
\text { ct-3' }\end{array}$ & $\begin{array}{l}23 \\
23\end{array}$ & 382 \\
\hline
\end{tabular}

Table S1: List of primers used to amplify 17 genetic variants for the multilocus assay

${ }^{\dagger} \mathrm{F}$ - forward primer; $\mathrm{R}$ - reverse primer; bp - base pair; Primer sequences in bold are unrelated sequences added to the 5 ' end of the primer to enable multiplexing. 
SUPPLEMENTARY TABLE S2:

\begin{tabular}{|c|c|c|c|c|}
\hline & \multicolumn{2}{|c|}{ MPCR-A } & \multicolumn{2}{|c|}{ MPCR-B } \\
\hline Reagents & $\begin{array}{l}\text { Final } \\
\text { Concentration }\end{array}$ & $\begin{array}{l}\text { Volume per } \\
\text { reaction }(\mu \mathrm{L})\end{array}$ & $\begin{array}{l}\text { Final } \\
\text { Concentration }\end{array}$ & $\begin{array}{l}\text { Volume per } \\
\text { reaction }(\mu \mathrm{L})\end{array}$ \\
\hline $\begin{array}{l}\text { 2X Taq Master } \\
\text { mix }\end{array}$ & $1 \mathrm{X}$ & 25.0 & $1 \mathrm{X}$ & 25.0 \\
\hline $\begin{array}{l}\text { Primer Master } \\
\text { mix } \\
\text { A / B }(2 \\
\text { pmole } / \mu \mathrm{L})^{\dagger}\end{array}$ & 10.0 pmole & 5.0 & 15.0 pmole & 7.5 \\
\hline Q-solution (5X) & $0.5 \mathrm{X}$ & 5.0 & $0.5 \mathrm{X}$ & 5.0 \\
\hline Distilled water & - & 12.0 & - & 9.5 \\
\hline$\overline{D N A}$ & $\approx 900 \mathrm{ng}$ & 3.0 & - & 3.0 \\
\hline \multicolumn{2}{|l|}{ Total volume } & 50.0 & & 50.0 \\
\hline \multicolumn{5}{|c|}{ PCR Conditions } \\
\hline \multicolumn{5}{|c|}{$\begin{array}{l}95^{\circ} \mathrm{C} \text { for } 15 \text { mins (Initial denaturation); } 95^{\circ} \mathrm{C} \text { for } 1 \mathrm{~min} \text { (denaturation); } 60^{\circ} \mathrm{C}(\mathrm{MPCR}-\mathrm{A}) \text {, } \\
55^{\circ} \mathrm{C} \text { (MPCR-B) for } 2 \text { mins (annealing); } 72^{\circ} \mathrm{C} \text { for } 2 \text { mins (Elongation) followed by } 32 \\
\text { cycles \& final elongation at } 72^{\circ} \mathrm{C} \text { for } 10 \text { mins. }\end{array}$} \\
\hline
\end{tabular}

Table S2: Master Mix Preparation of MPCR-A / -B along with the PCR cycling condition

${ }^{\dagger}$ The respective primer master mix (A/B) was prepared by aliquoting $10 \mu \mathrm{L}$ (stock concentration $100 \mathrm{pmole} / \mu \mathrm{L}$ ) of each of primer pair (forward \& reverse) in a total volume of $500 \mu \mathrm{L}$ (final concentration 2 pmole $/ \mu \mathrm{L})$. 


\section{SUPPLEMENTARY TABLE S3:}

\begin{tabular}{|c|c|c|c|c|c|}
\hline No. & $\begin{array}{l}\text { Gene name } \\
\text { (Variants) }\end{array}$ & $\begin{array}{c}\text { Gene } \\
\text { ID }\end{array}$ & $\begin{array}{l}\text { Allele specific oligonucleotide Probe } \\
\text { Sequences } \dagger \\
\left(5^{\prime}-3^{\prime}\right)\end{array}$ & $\begin{array}{l}\text { Probe } \\
\text { length } \\
\text { (bases) }\end{array}$ & $\begin{array}{c}\text { Probe } \\
\text { concentration } \\
\text { (picomoles) }\end{array}$ \\
\hline 1 & $\begin{array}{c}\text { LDLR } \\
\text { (ins242G) }\end{array}$ & 3949 & $\begin{array}{l}\text { WT: amino-5' -act gag gaa tgc agc ggt tga-3' } \\
\text { MT: amino-5' -act gag gaa tgc age cgg ttg } \\
\text { ac-3' }\end{array}$ & $\begin{array}{l}21 \\
23\end{array}$ & $\begin{array}{l}15.0 \\
1.0\end{array}$ \\
\hline 2 & $\begin{array}{c}L D L R \\
(\text { ins397G) }\end{array}$ & 3949 & $\begin{array}{l}\text { WT: amino-5' -cgt cca agc agt cec ggt c-3' } \\
\text { MT: amino-5' -cgt cca agc agt ccc cgg tc-3' }\end{array}$ & $\begin{array}{l}19 \\
20\end{array}$ & $\begin{array}{l}75.0 \\
15.0\end{array}$ \\
\hline 3 & $\begin{array}{c}\text { LDLR } \\
(\mathrm{E} 387 \mathrm{~K})\end{array}$ & 3949 & $\begin{array}{l}\text { WT: amino-5' -tca cca acc ggc acg agg tc-3' } \\
\text { MT: amino-5' -tca cca acc ggc aca agg tc-3' }\end{array}$ & $\begin{array}{l}20 \\
20\end{array}$ & $\begin{array}{l}300.0 \\
160.0\end{array}$ \\
\hline 4 & $\begin{array}{c}\text { LDLR } \\
(\mathrm{L} 393 \mathrm{~K})\end{array}$ & 3949 & $\begin{array}{l}\text { WT: amino-5' -agg aag atg acg ctg gac cg- } \\
\text { 3' } \\
\text { MT: amino-5' -agg aag atg acg cgg gac cg- } \\
\text { 3', }\end{array}$ & $\begin{array}{l}20 \\
20\end{array}$ & $\begin{array}{l}2000.0 \\
1200.0\end{array}$ \\
\hline 5 & $\begin{array}{c}L P L \\
\text { (D9N) }\end{array}$ & 4023 & $\begin{array}{l}\text { WT: amino-5'-ttt act ttc gat gtc gat aaa atc } \\
\text { tc-3' } \\
\text { MT: amino-5'-ttt act ttc gat gtt gat aaa atc } \\
\text { tc-3' }\end{array}$ & $\begin{array}{l}26 \\
26\end{array}$ & $\begin{array}{l}20.0 \\
40.0\end{array}$ \\
\hline
\end{tabular}




\begin{tabular}{|c|c|c|c|c|c|}
\hline 6 & $\begin{array}{c}L P L \\
(\mathrm{~N} 291 \mathrm{~S})\end{array}$ & 4023 & $\begin{array}{l}\text { WT: amino-5'-ggc tat gag atc aat aaa gtc } \\
\text { ag-3' } \\
\text { MT: amino-5'-ggc tat gag atc agt aaa gtc } \\
\text { ag-3' }\end{array}$ & 23 & $\begin{array}{l}85.0 \\
85.0\end{array}$ \\
\hline 7 & $\begin{array}{c}\text { CETP } \\
(\mathrm{I} 405 \mathrm{~V})\end{array}$ & 1071 & $\begin{array}{l}\text { WT: amino-5'-gca get ccg agt ccg tcc ag-3' } \\
\text { MT: amino-5'- gca gct ccg agt cca tcc ag-3' }\end{array}$ & $\begin{array}{l}20 \\
20\end{array}$ & $\begin{array}{l}60.0 \\
250.0\end{array}$ \\
\hline 8 & $\begin{array}{c}\text { CETP } \\
(\mathrm{D} 442 \mathrm{G})\end{array}$ & 1071 & $\begin{array}{l}\text { WT: amino-5'-gtg agc ctc ttc gac atc atc-3' } \\
\text { MT: amino-5'-gtg agc ctc ttc ggc atc atc-3' }\end{array}$ & $\begin{array}{l}21 \\
21\end{array}$ & $\begin{array}{l}10.0 \\
2.0\end{array}$ \\
\hline 9 & $\begin{array}{c}\text { LIPC } \\
(\mathrm{C}-514 \mathrm{~T})\end{array}$ & 3990 & $\begin{array}{l}\text { WT: amino-5'-acc ctt cac cce cgt gtc-3' } \\
\text { MT: amino-5'-acc ctt cac ccc cat gtc-3' }\end{array}$ & 18 & $\begin{array}{l}350.0 \\
500.0\end{array}$ \\
\hline 10 & $\begin{array}{c}F G B \\
(\mathrm{G}-455 \mathrm{~A})\end{array}$ & 2244 & $\begin{array}{l}\text { WT: amino-5'-ggg cca tta aaa tea ata gta } \\
\text { atg-3' } \\
\text { MT: amino-5' -ggg cca tta aaa tta ata gta } \\
\text { atg-3' }\end{array}$ & $\begin{array}{l}24 \\
24\end{array}$ & $\begin{array}{l}20.0 \\
30.0\end{array}$ \\
\hline 11 & $\begin{array}{l}I T G B 3 \\
\text { (L33P) }\end{array}$ & 3690 & $\begin{array}{l}\text { WT: amino-5'-gtg agc cca gag gca ggg c-3' } \\
\text { MT: amino-5'-gtg agc ccg gag gca ggg c-3' }\end{array}$ & $\begin{array}{l}19 \\
19\end{array}$ & $\begin{array}{l}25.0 \\
15.0\end{array}$ \\
\hline 12 & $\begin{array}{c}A G T \\
(\mathrm{M} 235 \mathrm{~T})\end{array}$ & 183 & $\begin{array}{l}\text { WT: amino-5'-ctg get get cce tga tgg gag } \\
\text { cc-3' } \\
\text { MT: amino-5'-ctg gct get cce tga } \mathbf{c g g} \text { gag }\end{array}$ & 23 & $\begin{array}{r}900.0 \\
1000.0\end{array}$ \\
\hline
\end{tabular}




\begin{tabular}{|c|c|c|c|c|c|}
\hline & & & cc-3' & & \\
\hline 13 & $\begin{array}{r}\text { AGTRl } \\
\text { (A1166C) }\end{array}$ & 185 & $\begin{array}{l}\text { WT: amino-5'-cac tac caa atg agc att agc } \\
\text { tac-3' } \\
\text { MT: amino-5'-cac tac caa atg agc } \underline{\mathbf{c t t}} \text { agc } \\
\text { tac-3' }\end{array}$ & $\begin{array}{l}24 \\
24\end{array}$ & $\begin{array}{l}200.0 \\
180.0\end{array}$ \\
\hline 14 & $\begin{array}{c}\text { NOS3 } \\
\text { (T-786C) }\end{array}$ & 4846 & $\begin{array}{l}\text { WT: amino-5'-ttc cet ggc tgg ctg acc c-3' } \\
\text { MT: amino-5'-ttc cct ggc } \text { cgg ctg acc c-3' }\end{array}$ & $\begin{array}{l}19 \\
19\end{array}$ & $\begin{array}{l}100.0 \\
275.0\end{array}$ \\
\hline 15 & $\begin{array}{c}\text { NOS3 } \\
\text { (E298D) }\end{array}$ & 4846 & $\begin{array}{l}\text { WT: amino-5'- ccc cag atg agc ccc cag aa- } \\
3 \text { ' } \\
\text { MT: amino-5'-ccc cag atg atc ccc cag aa-3' }\end{array}$ & $\begin{array}{l}20 \\
20\end{array}$ & $\begin{array}{l}325.0 \\
375.0\end{array}$ \\
\hline 16 & $\begin{array}{c}C B S \\
(\mathrm{~T} 833 \mathrm{C})\end{array}$ & 875 & $\begin{array}{l}\text { WT: amino-5' -tct gca gat cat tgg ggt gga tc- } \\
\text { 3' } \\
\text { MT: amino-5' -tct gca gat cac tgg ggt gga tc- } \\
\text { 3' }\end{array}$ & $\begin{array}{l}23 \\
23\end{array}$ & $\begin{array}{l}40.0 \\
40.0\end{array}$ \\
\hline 17 & $\begin{array}{c}M T R \\
\text { (A2756G) }\end{array}$ & 4548 & $\begin{array}{l}\text { WT: amino-5'-tta gac agg acc att atg ag-3' } \\
\text { MT: amino-5'-gat tag aca ggg cca tta tga g- } \\
\text { 3' }\end{array}$ & $\begin{array}{l}20 \\
22\end{array}$ & $\begin{array}{l}1500.0 \\
200.0\end{array}$ \\
\hline
\end{tabular}


Table S3: Allele specific oligonucleotide probe sequences and concentrations used for the hybridization

${ }^{\dagger}$ WT: wild type probe; MT: mutant type probe; Bold and underlined letter in the probe sequence indicates the nucleotide change; C6-amino group was labeled to the 5' end of each probe. 


\section{SUPPLEMENTARY FIGURE S4}

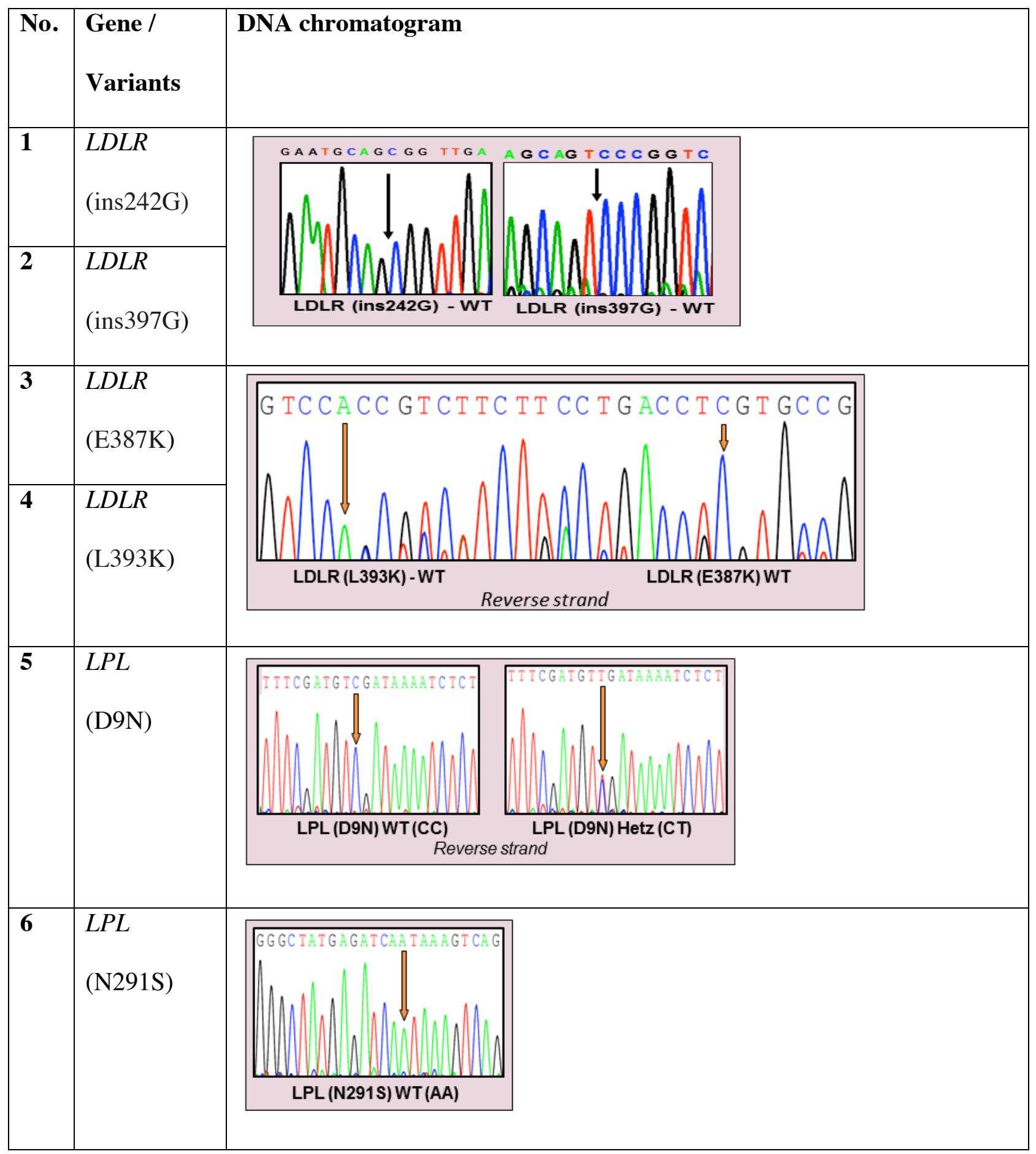




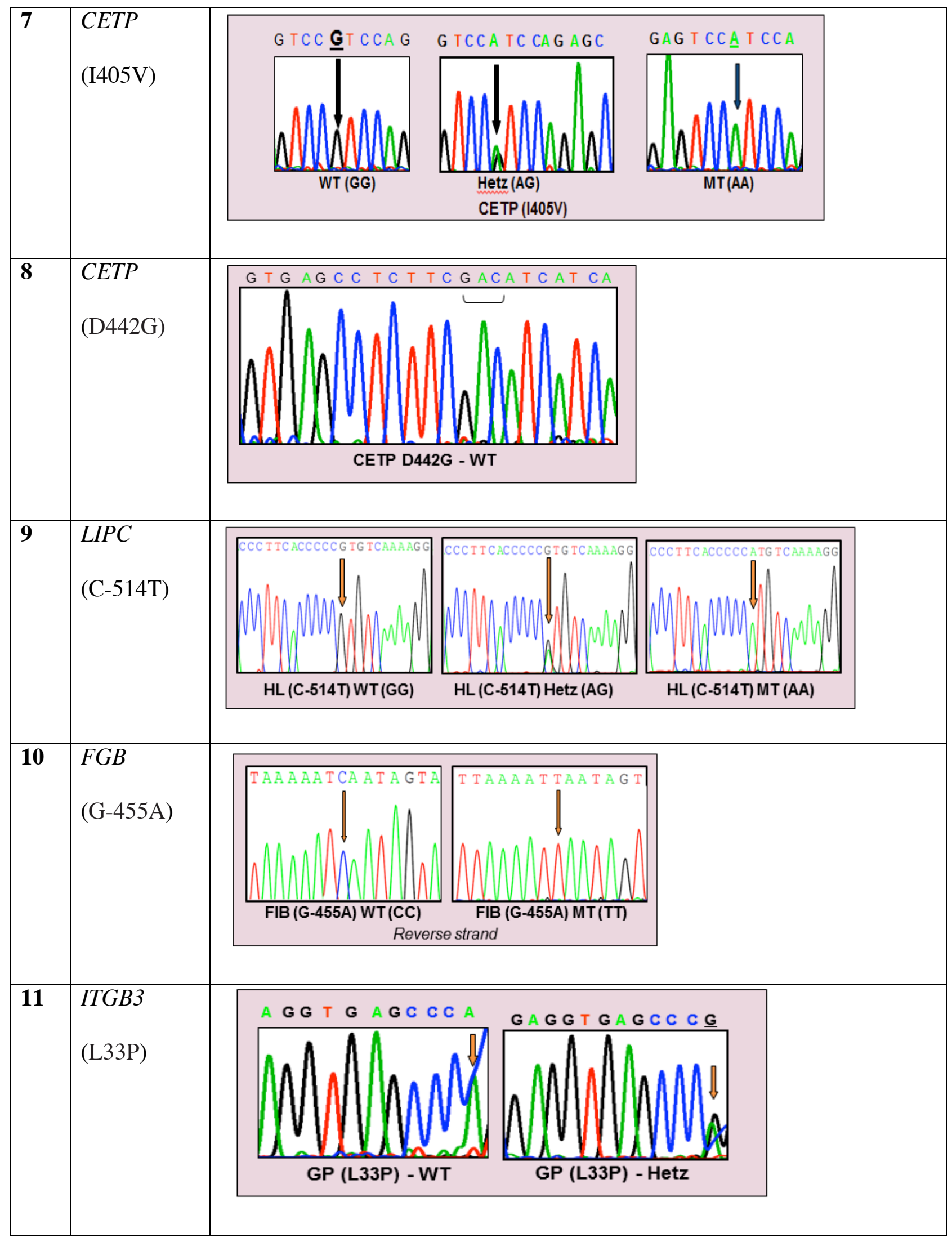




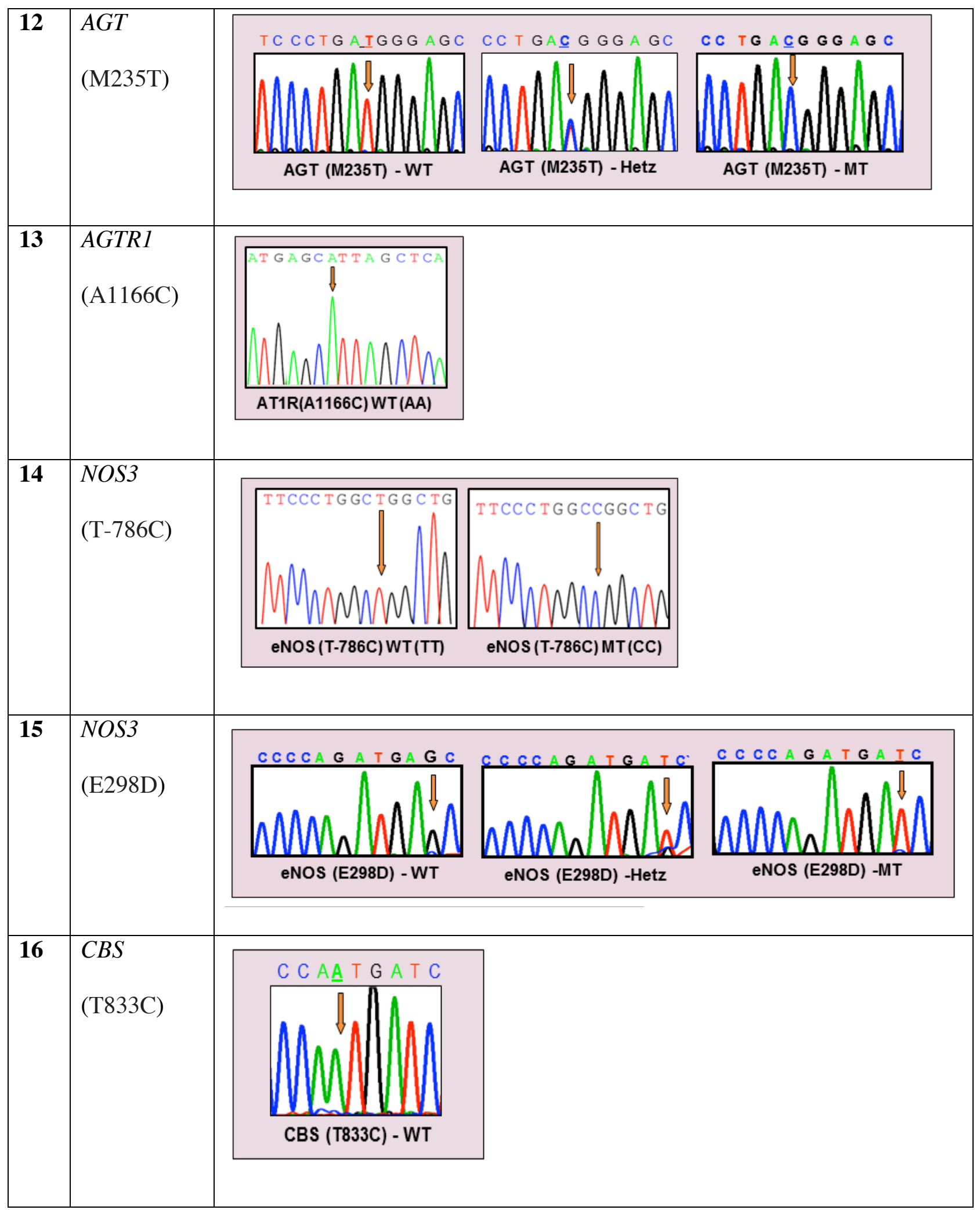




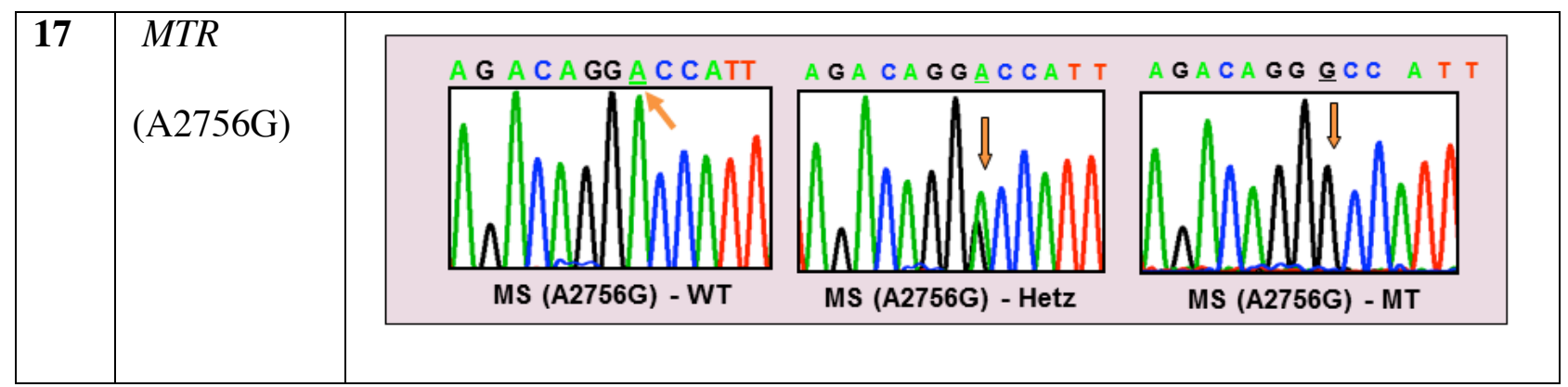

Figure S4: Representative chromatogram for each of the 17 genetic variant in the study 